\title{
Historia sucinta de las hemorroides
}

\author{
Concise history of hemorrhoids
}

\author{
Luis Guerra Montero ${ }^{\mathrm{a},{ }^{*}}$ \\ a Hospital Regional Docente de Enfermedades Neoplásicas, Huancayo, Perú
}

\section{RESUMEN}

Las hemorroides representan la patología más frecuente de las enfermedades ano-rectales benignas que son tratadas por médicos gastroenterólogos y cirujanos con entrenamiento especial en proctología que es una rama de la medicina especializada en el diagnóstico y tratamiento de las enfermedades del ano y recto, su nombre deriva del griego haema (sangre) y rhoos (flujo). Dentro de todos los males que puede sufrir el hombre, la enfermedad hemorroidal sin lugar a dudas desempeña un papel importante en la vida humana tanto por los síntomas tan molestos que provoca -lo cual limita el desempeño social y cultural del hombre en la sociedad- como por la decisiva influencia que esta enfermedad ha ejercido en la historia de la humanidad ya que las hemorroides han afectado en diferentes épocas a reyes, gobernantes, líderes y pensadores de potencias mundiales que debido a sus dolorosos síntomas o complicaciones de una manera $u$ otra manera han decidido el futuro de sus naciones y del mundo entero. Así mismo su diagnóstico y tratamiento implico el desarrollo necesario de la medicina artesanal poco científica y quizás dolorosa en sus inicios a una medicina moderna nutrida de conocimientos actuales y avances tecnológicos muchas de las veces indolora como generalmente es hoy en día. Por todo ello consideramos que el conocimiento de la historia de las hemorroides es necesario para los médicos, enfermeras, historiadores, estudiantes y gente común y corriente.

\begin{abstract}
Hemorrhoids are the most common pathology of anorectal benign diseases, which are treated by gastroenterologists and surgeons with special training in proctology, which is a branch of specialized medicine in the diagnosis and treatment of the anus and rectum diseases, its name derived from the Greek Haema (blood) and rhoos (flow). Among all the human ailments, undoubtedly hemorrhoidal disease plays an important role in human life because of bothersome symptoms it causes-which limits social and cultural performance of human in society, also the decisive influence, that this disease has had in history of mankind, hemorrhoids have affected at different times kings, rulers, leaders and thinkers of first world countries, because of their painful symptoms or complications in one way or another have decided the future of their nations and the world. Likewise its diagnosis and treatment implied the necessary development of folk unscientific medicine and perhaps painful in its early beginning to modern medicine nourished of current knowledge and technological advances many times painless as It's usually today. Therefore we believe that the knowledge of the history of hemorrhoids is necessary for doctors, nurses, historians, students and common people.
\end{abstract}

Keywords: History, hemorrhoidal disease, folk medicine, modern medicine.

Palabras clave: Historia, enfermedad hemorroidal, medicina artesanal, medicina moderna.

Historial del artículo:

Recibido, 7 de marzo de 2016; aceptado, 14 de marzo de 2016; disponible en línea, 30 de marzo 2016

* Doctor en Medicina, Especialista en Gastroenterología.

Correo:guerramontero@gmail.com

Este es un artículo de acceso abierto, licencia CC BY (https://creativecommons.org/licenses/by/4.0/) 


\section{INTRODUCCIÓN}

El término Hemorroides viene del griego haema (sangre) y rhoos (flujo) su historia data desde tiempos muy remotos, prácticamente con la aparición del hombre sobre la faz de la tierra. Las hemorroides, prolapsos, fístulas y abscesos anales, han afectado en diferentes épocas de la historia, a reyes, gobernantes, líderes, pensadores y gente común, el hombre estuvo a merced de ellas hasta que se desarrollaron las terapéuticas actuales.

\section{Mundo antiguo}

Los primeros registros que nombran a las hemorroides se remontan hacia el año 2200 antes de cristo y proceden del Código de Hammurabi (Rey de Babilonia), donde se fijan los honorarios de los Proctólogos (1) lo que "nos hace pensar que estos ya eran muy importantes en esa época y la enfermedad preocupaba de sobremanera a los habitantes de aquel entonces".

Luego en papiros de Egipto de 1500 años antes de cristo a las hemorroides se las identifican como una protrusión rectal y según Herodoto en esta cultura la medicina estaba muy adelantada a tal punto que cada médico estaba especializado en tratar una sola enfermedad por lo cual existían médicos que trataban solo hemorroides algunos de los cuales usaban el pomposo título de "Oculista y guardián del ano del faraón" (2).

En Grecia durante su "época de oro" 500-350 años antes de cristo las hemorroides eran consideradas un castigo de los dioses, Hipócrates en su obra Corpus hipocráticus mencionaba "Estas venas al calentarse, atraen la sangre de las venas cercanas e ingurgitándose por dentro del intestino se inflaman exteriormente, con cabezas salientes que son raspadas al salir por las heces, lesionándose y arrojando sangre a chorros..." (3). Galeano en el año 131 antes de cristo en su libro de Las Epidemias nos dice que "Las hemorroides, lo mismo que las varices, disipan los sufrimientos de la gota y los dolores de las articulaciones" (2).

En los Evangelios es conocido el episodio de la mujer "hemorroidosa" que curó Jesucristo y en el libro de Samuel aparecen referencias concretas acerca de las hemorroides, consideradas como una consecuencia de la ira de Dios contra los Filisteos por haber robado el Arca de la Alianza tras su victoria contra los judíos (2).

Cabe resaltar que en esos tiempos las hemorroides se trataban con hierros candentes, dice Hipócrates "Yo recomiendo preparar siete $\mathrm{u}$ ocho pedazos de hierro, tamaño de una brasa y una espina hueca gruesa que en un extremo tiene un gancho y en el otro un pequeño recipiente oval, el día anterior al procedimiento purgar al enfermo con medicamento y el día de la operación colocar al enfermo sobre su espalda sobre una almohada en su cadera, luego con los dedos exteriorizar hasta el ano tanto como sea posible las hemorroides, aplicar el cauterio hasta secarlas sin omitir ninguna, luego aplicar una cataplasma de lentejas y brea finamente trituradas y hervidas por cinco o seis días, al séptimo día colocar una gasa humedecida con miel en el ano tan profundo como sea posible y dejarla dentro..." (3).

\section{Época medieval}

Aetius de Armida (502-575) en su obra "Tetrabiblon" destaca las primeras operaciones para hemorroides de Oribasius realizadas con espejos cilíndricos cónicos y valvulas de varias valvas. Paulus de Aegina (625690), "padre de la medicina islámica" en sus "Siete libros de medicina", perfecciono las ideas y técnicas de Galeno e incorporó otras griegas, sirias y árabes para el tratamiento de las hemorroides. En los años venideros 800, 900 después de cristo los textos de cirugía proctológica más importantes halladas en la biblioteca de Carlomagno se limitaban a copias de fragmentos de "Curandis Morbis" de Serenus Sammonicus, partes del Corpus Hipocraticus de Hipócrates y obras de Celsius, Galeno, Soranus, Aetius y Paulus de Aegina (3).

A inicios del año 1000 las operaciones para hemorroides fueron difundidas en Salerno por el monje Constantine Africanus (1020-1087), dando inicio así a la creación de las primeras escuelas de medicina moderna, luego se crearían la escuela de Boloña (1119), de París (1150) y la de Oxford (1170) en todas ellas se practicaban las técnicas quirúrgicas mejoradas de Hipócrates, Galeano, Celsius y Dioscorides (4).

Más adelante los progresos en los procedimientos quirúrgicos, fueron llevados a cabo por los médicos europeos llamados "los cirujanos principales", tales como Lanfrank de Milano (1250-1306), Guy de Chauliac (1300-1368), Enrique de Mondeville (1260-1320) y Jhon de Ardene (1307-1370) quienes ampliaron y refinaron los procedimientos quirúrgicos para la hemorroides existentes hasta ese momento (5).

Aunque por esas años se publicaron los primeros textos que se referían al tema como la "Chirurgia Magna", (París 1296), "Chirurgia" (Paris 1363), que fue el texto de imprescindible consulta durante tres siglos, con 34 ediciones en manuscrito y 70 impresas a partir de 1478 (3) el progreso de la ciencia y de la cirugía proctológica se atascó por cerca de 350 años donde se remplazó el termino de hemorroides por el de "emorides", "amouranes", "amourrances", "amereaudes", etc. y estos términos se refieren más al conjunto de afecciones anales que al propio proceso 
hemorroidal en sí (2).

En esta misma época San Fiacro (Fiacre en francés, Fiachra en celta), patrón de los jardineros, se convierte en el patrón de los enfermos de hemorroides y esta enfermedad es llamada "el mal de San Fiacro". La leyenda cuenta que san Fiacro en su celo para obtener la máxima cantidad de tierra cultivable trabajaba durante todo el día lo cual a la larga le produjo un caso de prolapso hemorroidal, el mismo creyendo que su fe lo podía curar se sentó en una gran piedra y pidió a Dios para un alivio milagroso y al cabo de una horas se dio con la sorpresa que su problema había sido curado sin embargo la imagen de sus hemorroides quedo grabada en aquella piedra ihasta el día de hoy muchas enfermos de todo el mundo continúan sentándose en esa piedra legendaria con la esperanza de conseguir alivio a sus molestiasi (6).

También se dice que Enrique $V$ de Inglaterra (14131422) murió en Vincennes de la enfermedad de San Fiacro ya que no quería operarse y se limitaba solo hacer plegarias al santo para su pronta curación sin embargo el milagro nunca se le realizo. En aquel entonces los métodos operatorios se basaban en la técnica de Aecio de Constantinopla («exteriorización de las hemorroides con un gancho y sección de las mismas con un cuchillo de ancha lámina») y el de aplastarlas y cauterizarlas con unas pinzas de hierro al rojo vivo. No es de extrañar, pues que Enrique $V$ prefiriese rogarle al Santo antes de someterse a tan dolorosa operación (2).

\section{Renacimiento y Siglo XVIII}

En el Renacimiento la cultura médica no podía estacionarse frente al desarrollo científico que caracterizó dicha época de entre todos los médicos que contribuyeron al mejor conocimiento de la patología hemorroidal destaco Ambrosio Paré (15101592) que desterró el aforismo hipocrático de que "las enfermedades no curables por el hierro se curan por el fuego». Andres Vesalio (1514-1564) que legó una detallada información de la anatomía del recto y del colon, con unos grabados en madera que se conservan hasta la actualidad (7).

Giovanni Batista Morgagni (1682-1771) que además de las hemorroides describió las otras principales patologías anorrectales. Jerome Fabrice D'Acquapendente (1537-1619) considerado por algunos autores el más grande proctólogo del Renacimiento; escribió "Opera Chirurgica", en donde informa de nuevos métodos para el tratamiento de las hemorroides con instrumentos quirurgicos modificados por el mismo. Jean Louis Petit (16741750) que en su "Traité des maladies chirugicales et des operations" indico que la cirugía de hemorroides llamada "incisional sola" causa mayor dolor por la sensibilidad de la piel del ano y tiene mayor posibilidad de hemorragias fatales (7).

Lorenz Heister (1683-1758) fue el primero en describir un procedimiento detallado para la ligadura refería que "Se debe atar los hemorroides de la sangría con una aguja e hilo, luego se deben cortar las piezas que se dilaten más allá de la ligadura, teniendo cuidado en dejar las venas más pequeñas pues podían abrirse por largo tiempo...". Aunque inicialmente hubo una reticencia respecto a este nuevo tratamiento puesto que se creía que podía producir más dolor y gangrena con los años se demostró que las ligaduras solo causaban molestias inespecíficas y una leve inflamación dado que la mucosa rectal no tenía la sensibilidad de la piel anal (8).

En este periodo también es interesante mencionar la importancia histórica de la enfermedad hemorroidal de Napoleón Bonaparte (1769-1821). Se dice que el Emperador en la mañana de la batalla de Waterloo tenía un intenso dolor anal razón por la cual no pudo montar a caballo hasta después de las 10, una hora inhabitual en él y demasiado tarde para dirigir personalmente y enderezar el curso de la batalla que la estaba perdiendo que a la postre fue el final de su imperio y el inicio de una nueva etapa en toda Europa $(2,3)$.

Otros famosos enfermos fueron Lutero, que escribió sobre sus hemorroides; Don Juan de Austria, el vencedor de Lepante, murió en Namur a consecuencia de una hemorragia rectal, cuatro horas después de haber sido operado de hemorroides. El zar de Rusia Pedro III parece que murió también víctima de una "fuerte crisis hemorroidal".

\section{El Hospital St. Mark's}

Pero no fue sino hasta el Siglo XIX donde se empieza a tener conocimientos más profundos de la patología hemorroidal así como de nuevas técnicas quirúrgicas y esclerosantes impuestas principalmente por Frederick Salmon $(1796-1,898)$ pionero de la cirugía anorrectal de ese siglo que undo el "St. Mark's Hospital for Fístula and other Diseases of the Rectum» en Londres en el año 1835 para "los pobres que sufrían hemorroides, fístulas y otras enfermedades del recto», al principio era una pequeña habitación en Aldergate Street número 11, y allí estuvo durante tres años, luego se trasladó a la 38 de Charterhouse Square. En Aldergate había 7 camas, y el primer año ya hubo 131 enfermos internados, en Charterhouse dispuso de 14 camas, y allí estuvo 13 años hasta que se trasladó a City Road, en donde sigue hasta el día de hoy $(2,9)$.

Esta institución demuestra lo que puede hacer la fe y la constancia de un hombre, porque Salmón tuvo que soportar muchas oposiciones y críticas, y trabajó 


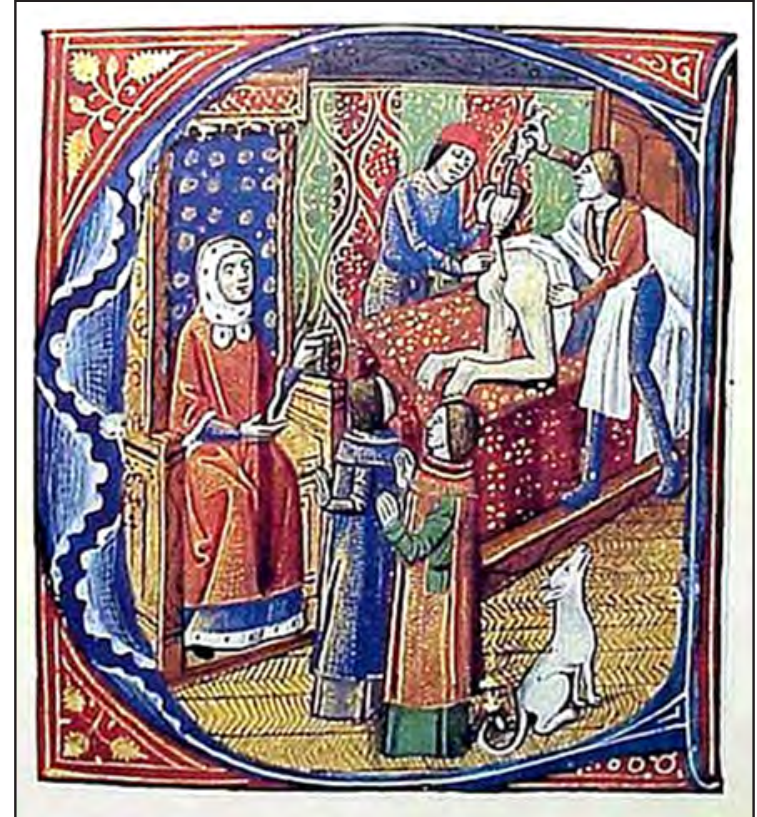

Figura $\mathrm{N}^{\circ}$ 1: Hemorroides, versión enema

Fuente: (http://historiasde lahistoria.com/2010/11/24/ írias-al-medico-en-la-edad-media).

sólo durante unos veinte años. Cuando se retiró, en 1859, se dijo de él que no sólo era un hard worker "gran trabajador", sino también un lucky surgeons "cirujano afortunado", pues tenía en su haber 3500 operaciones sin un solo resultado fatal. Claro que hay que suponer que muchas de estas operaciones serían menos graves que las actuales. El Hospital lo fundó a los 39 años y se retiró a los 63, murió a los 72 años. Escribió asimismo "Practical Treatise on Stricture of the Rectum" un clásico para todos los que nos interesa la proctologia $(2,9)$.

\section{Siglo XX}

La evolución terapéutica y quirúrgica del siglo XX evidencia grandes progresos; destacándose entre ellos, la técnica de Milligan y C. Naughton Morgan en 1935 llamada "Open Hemorrhoidectomy" consiste en el corte de piel perianal, disección del plexo hemorroidal de los músculos anales y ligadura de las hemorroides, que es la más usada en el Reino Unido y en Europa. En 1959 D. Ferguson y J. Heaton, en los Estados Unidos aportaron una modificación a la técnica de MilliganMorgan en la que las incisiones son cerradas parcial o totalmente. "Close hemorrhoidectomy" muy popular en los Estados Unidos (3).

En 1998 Longo describió la "Hemorroidectomia con grapas" a través de un instrumento que lleva su nombre que consiste básicamente en corte y "grapado" circular transanal de la mucosa anorrectal sobrante mediante un instrumento. Sin embargo hoy en día las alternativas medicas no quirúrgicas son las que cuentan mayor popularidad tanto por su altísima efectividad, menor efectos adversos -dolor- y menor costo, entre ellas tenemos "Ligadura con bandas elásticas" de Barron (1963), "Fotocoagulación infrarroja", de Leicester RJ, Nicholls RJ (1981), y "Escleroterapia y coagulación bicap" de Barwell J, Watkins RM (1999) $(3,10)$.

Actualmente la mayoría de estas técnicas son usadas, decidiéndose su elección en conjunto con el paciente dependiendo en gran medida del grado de afectación y de las complicaciones que pudieran presentar posteriormente. Una anécdota reciente sobre las hemorroides la protagonizo el futbolista Leonardo Ponzio del club River Plate que el 2012 juego todo el clásico argentino con su eterno rival Boca Junior aquejado de un intenso sangrado (que lo obligo a cambiarse varias veces de pantalón) provocada por una hemorroide interna trombosada. Al finalizar el partido los periodistas deportivos lo cuestionaron de porque no había abandonado la cancha para que entrara otro jugador con mejor estado físico a lo cual él respondió con uno frase que lo inmortalizo y quedo grabado en la hinchada del cuadro millonario "Pensé que se iba a cortar además si nos empataban la foto de mi pantalón sangrado iba a estar en todos los afiches de Boca y eso no lo hubiera permitido jamás" (11).

\section{CONCLUSIONES}

Hoy en día la enfermedad hemorroidal es tratada en todo el mundo y tal vez sean Francia, Inglaterra y Estados Unidos los países donde han nacido los más brillantes proctólogos que contribuyeron a conocer, diagnosticar y tratar esta patología aunque afortunadamente para el mundo entero en todas partes han existido y existen magnificas escuelas de proctología con destacados gastroenterólogos y cirujanos que pasaron de usar los hierros candentes a ligaduras con bandas elásticas, de usar espejos cilíndricos cónicos y válvulas de varias valvas a instrumentos que hacen mínimos cortes y grapas en las cirugías.

También podemos mencionar que la enfermedad hemorroidal es una de las pocas patologías que ha tenido real influencia en el desarrollo de la historia de la humanidad, cual habría sido el desenlace de la guerra entre Inglaterra y Francia si el valiente Enrique $\checkmark$ no hubiera fallecido, que hubiera ocurrido con Europa si Napoleón Bonaparte se hubiera presentado a primera hora en Waterloo y ganaba aquella mítica batalla, lastimosamente nunca lo sabremos.

Finalmente cabe resaltar que la medicina se debe a su pasado, lo que aconteció en tiempos remotos condicionaron el futuro, determinaron las líneas maestras a seguir en el desarrollo de las nuevas técnicas de diagnósticos y tratamientos de las diversas 
enfermedades y en este caso en particular de la enfermedad hemorroidal.

\section{REFERENCIAS \\ BIBLIOGRÁFICAS}

1. Karchmer S. Códigos y juramentos en medicina. Acta Médica Grupo Ángeles. 2012; 10(4): 224 234.

2. Fernández G. Historia sucinta de la proctología. Santiago de Compostela; 2009. Disponible en: http://files.sld.cu/coloproctologia/files/2009/1 1/ historia_de_la_proctologia.pdf

3. Manzanilla M. Historia de las hemorroides y su tratamiento quirúrgico. Revista Mexicana de Coloproctología. 2005; 11(1): 4-7.

4. Tamayo C. El aporte cultural y educativo de la Baja Edad Media. Educación y educadores. 2007; 10(2): 197-213.

5. Gómez OL, López JE. Historia de la cirugía en la época pre-Razetti. Colección Razetti. 2005; I: 211-343.

6. Ganz R. The Evaluation and Treatment of Hemorrhoids: A Guide for the Gastroenterologist. Clinical Gastroenterology and Hepatology. 2013; 11 (6): 593-603.

7. Garrison FH. Introducción a la historia de la medicina. 3 ed. Madrid: WB Saunders Company Philadelphia; 1921.

8. Martinez G. Reyes H. McBurney: the incision, the point, and the surgeon Cir Gen 2007; 29:70-75

9. Dukes CE. Frederick Salmon: Founder of St. Mark's Hospital, London. Med Hist. 1959; 3(4): 312-316.

10. Fernandez G. Historia sucinta de la proctologia en Lentini J Temas de Proctologia. Barcelona: Fontalba; 1982.

11. Massobrio F. Ponzio: "Si empatábamos, esta foto iba a estar en los afiches de Boca". La Nación. 11 de junio de 2012. Disponible en: http:// canchallena.lanacion.com.ar/1480955 